\title{
The Benefits of Prenatal Aromatherapy Massage and Prenatal Yoga in Improving Beta Endorphin and Alleviating Back Pain for Pregnant Women
}

\author{
*1 $1^{\text {st }}$ Sylvi Wafda Nur Amellia \\ Study Program of Midwifery and \\ Midwifery Education \\ Health Education Academy of \\ AKBIDYO \\ Yogyakarta, Indonesia \\ amellia.swn@gmail.com
}

\author{
$2^{\text {nd }}$ Riadini Wahyu Utami \\ Study Program of Midwifery and \\ Midwifery Education \\ Health Education Academy of \\ AKBIDYO \\ Yogyakarta, Indonesia \\ riadiniwahyutami@gmail.com
}

\begin{abstract}
Aggravating back pain during pregnancy can cause stress for pregnant women and hinder the growth of fetus. Yoga and aromatherapy back massage can help alleviate physiological back pain during pregnancy. The research determines the effectiveness of combined methods of aromatherapy back massage and prenatal yoga in improving beta endorphin and alleviating back pain for pregnant women. Sixty healthy primigravida women without any complication are divided into 4 groups $(n=15$ for each group), in the following arrangement: a control group which only participates in prenatal workout once a month and treatment groups which participate in prenatal workout once a week for 4 weeks, consisting of pregnant woman group attending prenatal yoga class and aromatherapy back massage, pregnant woman group attending only yoga class, and pregnant woman group attending only aromatherapy back massage. The level of beta endorphin in the blood plasma is measured after 4 treatment sessions. Back pain is measured using visual analogue scale (VAS). The difference between the scores of blood plasma and back pain following 4 treatment sessions in the four groups is determined using One-way Anova Post Hoc LSD Test. We have analysed 12 women from each group. The level of beta endorphin is significantly higher on each treatment group $(p<0,05)$. Back pain score significantly decreases in the observed group with p-value of 0,001 . The combination of prenatal yoga and aromatherapy back massage can significantly reduce the intensity of back pain and increase the level of beta endorphin. This research discovers the direct impact resulting in reduced back pain with an increased level of beta endorphin in groups receiving combined treatment in the forms of prenatal yoga and aromatherapy back massage classes.
\end{abstract}

Keywords - prenatal yoga, aromatherapy massage, back pain

\section{INTRODUCTION}

Pregnancy is a physiological process accompanied with the occurrence of both physical and psychological changes in pregnant women. Changes during pregnancy can cause discomfort for most pregnant women. Discomfort experienced by pregnant women typically varies between each trimester [1].

The period requiring specific treatment is the third trimester, considering in this period, fetus experiences a significantly higher rate of growth and development. One of the discomforts commonly experienced is low back pain, or more commonly known as LBP. This occurs due to a shift on body balance and the effect of walking for a significant period of time, resulting in tension and pain in the lumbar area [2].

Discomfort due to low back pain during pregnancy is disruptive to the daily activities. As many as $45 \%$ to $60 \%$ of pregnant women experiences mild low back pain and almost $10 \%$ of pregnant women experiences severe low back pain that hinders their activities [3]. Back pain is also caused by hormonal changes, musculoskeletal condition, cytokine inflammation, and oxidative stress. There are two hormones contributing to the stress and pain response, cortisol and beta endorphin. Beta endorphin is released into blood stream from the pituitary gland as a response to an episode of acute pain and stress, and it helps alleviate the pain. Cortisol is released as a response to chronic stress and chronic physiological and psychological condition, for instance pain and fear [4].

There are several ways implemented by healthcare workers in order to tackle various problems experienced by pregnant women, specifically those experiencing discomfort during the third trimester. These include pharmacological and non-pharmacological methods. One of the nonpharmacological methods is complementary and alternative medications (CAM), such as yoga, massage, chiropractic, relaxation technique, acupuncture, and acupressure, which are proven to be effective against back pain discomfort [5]. Non-pharmacological approaches are safer, simpler, and have fewer adverse side effects, as well as better suited for caregiving treatment for mothers, compared to pharmacological methods with various potential adverse effects. Additionally, complementary medications (Yoga, Massage, and Aromatherapy) influence pregnant women's psychological conditions as the relaxation effect caused can also positively impact the growth and development of fetus. Prenatal stress on mothers can negatively impact the health and safety of fetus as well as the mothers'. With that being said, during pregnancy and nearing childbirth, mothers require a peace of mind in order to avoid overwhelming anxiety. This is critical in ensuring pregnancy culminates in a healthy childbirth. 
In helping ensure the health of mothers, unborn babies, babies, and children, complementary medication becomes a subject of attention as a potential future-proof method for taking care of women during prenatal, perinatal, and postnatal period. Unfortunately, not all midwives implement complementary medication-based caregiving on pregnant women in order to help them alleviate low back pain[6].

As far as our current knowledge suggest, the effectiveness of aromatherapy and prenatal yoga has not been assessed or compared against. Pursuant to the aforementioned background, the researchers are interested in determining the benefits of prenatal aromatherapy massage and prenatal yoga in increasing beta endorphin and alleviating back pain in pregnant women. In practice, it is expected that the research can serve as an enriching literature on complementary therapy in the field of pregnancy caregiving, specifically regarding how to alleviate back pain.

\section{RESEARCH METHOD}

\section{A. Research Desaign}

The design for post-test only control group was adopted. Participants with pregnancy at 28-40 weeks attended prenatal yoga and/or aromatherapy back massage sessions once a week for 4 weeks. This research was conducted in four community health centres in Bantul between August and October of 2019.

\section{B. Participants}

Women with pregnancy at $28-40$ weeks were identified from patient lists and medical records. The evaluation for eligibility study was conducted between May and June of 2019. Participants' eligibility criteria were women with single pregnancy and who are deemed healthy enough for yoga class or massage by their midwives. Exclusion criteria for this research were women under the age of 20, those with a history of abortion, those with a history of cervical insufficiency, those diagnosed with placenta previa, or those prohibited from exercising due to cardiovascular condition or other complications. The researchers confirmed the eligibility of each participant by direct inquiry or from participants' midwives. Further, the researchers explained the research implications, schedule, and ethical consideration for each participant in writing.

\section{Research Schedule}

During the selection, written consent was obtained following researchers' explanation of this research. Further, participants filled questionnaire regarding demographic data, including occupation, last education, age, pregnancy age (in weeks), and health condition. After initial survey during selection, participants then attended yoga class or aromatherapy back massage once a week for 4 weeks. Assessment was conducted after participants attended 4 classes consecutively. Result assessment includes the measurement of back pain level, blood pressure and beta endorphin.

\section{RESULT VARIABLE AND IMPLEMENTATION PROTOCOL}

\section{A. Beta Endorphin Concentration}

$3 \mathrm{ml}$ of blood is sampled from each participant's brachialis blood vessel after 4 consecutive classes. Then, the blood samples are stored in phlebotomy tubes $5 \mathrm{ml}$ EDTA. Further, blood plasma is separated from blood through 10 minutes of centrifugation, following the retrieval of 500 microliters of plasma which is to be placed in micro-tubes. The level of beta endorphin is measured using ELISA-KIT E1291Hu Human Beta Endorphin B-EP Elisa Kit 96 T.

\section{B. Back Pain}

Back pain level is measured using Visual Analogue Scale (VAS) after all participants attend 4 consecutive classes. Back pain level is measured on a scale of 1 to 10 .

\section{Intervention}

Prenatal yoga and aromatherapy massage sessions are held in the community health centre once a week for 4 weeks. Researchers and yoga instructors hold the sessions in the community health centre. Yoga instructor is certified by Indonesia Yoga Association, which designs Hatha yoga program specifically for pregnant women. Each class takes 60 minutes (15 minutes of warm-up, 40 minutes of poses, and 5 minutes of meditation and breathing exercise). During warm-up, participants stretch their bodies while in sitting position. And then, they practice 7-8 yoga poses, followed with resting for 5 minutes, and practice another session of 78 yoga poses under the instructor's supervision. All poses are coordinated with breathing exercise. In order to avoid breathing problem, participants are asked to bend backward when inhaling and bending forward when exhaling in order to breathe more effectively. During meditation, all participants close their eyes while lying face-up or in lateral position, while meditating through picture guides provided by the instructor. Yoga is exercised on a yoga mattress, in a comfortable temperature and with relaxing music in the background.

Aromatherapy massage class is supervised by the researchers and counters. Back massage is administered using lavender aromatherapy oil from Young Living, mixed with virgin coconut oil (VCO) with a ratio of 1:5. Aromatherapy massage is re-administered for 30 minutes in a Sim's position. Participants are provided with souvenir at the end of each session.

\section{Ethical Consideration}

Research protocol has been approved by Ethics Committee of Ahmad Dahlan University of Indonesia (No. 021903002). Participants receive the explanation regarding the purpose and content of the research, voluntary nature of the participation, risk, how the exercise benefits pregnancy, and privacy consideration. Written approvals are obtained from the participants and acknowledged by the examining midwives.

During the research, health condition of each participant is monitored by the researchers and medical staffs using medical graphs. The researchers keep notes of participants' blood pressure and their conditions before and after the yoga 
sessions. Further, participants' fetus condition and heartbeat are examined using Doppler test before and after the class.

\section{E. Statistical Analysis}

The researchers compare the level of beta endorphin and back pain of all four groups after participating in 4 consecutive sessions using one-way ANOVA test. Prior to one-way ANOVA test, data normality is tested using Shapiro-Wilk test. In order to determine the respective differences between all four groups, LSD Post-Hoc analysis is performed. Statistical analysis is conducted using SPSS Statistics Version 17.0. For normality test, data is considered normally distributed when p-value is more than 0,05 . In this case, in one-way ANOVA test, data is considered statistically significant if $\mathrm{p}$-value is less than 0,05 .

\section{RESUlT}

This research is conducted in instances and for several respondent groups. Group I received prenatal yoga and aromatherapy massage treatment in the community health centre of Banguntapan II, Bantul. Group II received aromatherapy massage treatment in the community health centre of Kasihan. Group III received prenatal yoga treatment in Asih Waluyo Jati Clinic, Bantul. Group IV in community health centre of Sewon received no treatment (control group). Blood sampling was administered to all respondents to determine the level of beta endorphin and data regarding the level of back pain experienced by pregnant women in trimester III was examined.

Research result is analysed using SPSS for Windows. Normality test from the distribution of research result is conducted using Shapiro-Wilk test. The distribution of pregnant woman data sample following analysis is as follows:

TABLE I. DATA NORMALITY TEST WITH SHAPIRO-WILK ANALYSIS

\begin{tabular}{lccc}
\hline \multicolumn{1}{c}{ Observation group } & \multicolumn{2}{c}{ p value } & $\begin{array}{c}\text { Data } \\
\text { distribution }\end{array}$ \\
\cline { 2 - 3 } & Back pain & $\begin{array}{c}\text { Beta } \\
\text { endorphin } \\
\text { level }\end{array}$ & \\
\hline Control & 0.412 & 0.233 & Normal \\
Prenatal yoga & 0.102 & 0.292 & Normal \\
Massage & 0.333 & 0.780 & Normal \\
Prenatal yoga and massage & 0.570 & 0.375 & Normal \\
\hline
\end{tabular}

Table above indicates that the p-value of all groups is $>0,05$, meaning the sampling data is distributed normally, which meets the requirements of parametric test. Parametric test is performed using one-way ANOVA test to determine any variance to the sample group. The result of comparison test between groups is as follows:

TABLE II. BACK PAIN COMPARISON (\%)

\begin{tabular}{lcc}
\hline Observation group & $\begin{array}{c}\text { Means } \pm \text { standard } \\
\text { deviation }\end{array}$ & p-value \\
\hline Control & $6.08 \pm 1.37$ & $0.001<\alpha$ \\
Prenatal yoga & $4.75 \pm 1.49$ & \\
Massage & $4.45 \pm 1.44$ & \\
Prenatal yoga and massage & $3.42 \pm 1.68$ & \\
\hline
\end{tabular}

Table 2 on the result of several comparison tests using LSD test indicates that there is a significant difference in the level of back pain experienced by control group and treatment group. The average value indicates the decrease of pain experienced by the treatment group receiving combination treatment.

TABLE III. COMPARISON OF BETA ENDORPHIN LEVEL (\%)

\begin{tabular}{lll}
\hline Observation group & Means \pm standard deviation & p-value \\
\hline Control & $32.42 \pm 380$ & $0.04<\alpha$ \\
Prenatal yoga & $32.87 \pm 8.41$ & \\
Massage & $41.10 \pm 13.63$ & \\
Prenatal yoga and & $46.22 \pm 12.62$ & \\
massage & & \\
\hline
\end{tabular}

Table 3 indicates the average difference between the control group and the treatment group, where the $p$-value is less than 0,05 . This indicates the difference of beta endorphin level between pregnant woman group receiving combined treatment of prenatal yoga and aromatherapy massage and those who did not receive combination treatment.

To the extent of researchers' knowledge, there is no study that directly links the effect of combination treatment of prenatal yoga and aromatherapy massage on back pain experienced by pregnant women. Third Trimester pregnant women who practiced antenatal yoga. This study concludes that pregnant women who routinely practice antenatal yoga have fewer pregnancy problems[7].

In pregnancy, hormonal change triggers a reaction on the soft tissue supporting and connecting bones, causing reduced elasticity and flexibility on the muscles [8]. Posture change during pregnancy may be caused by weight gain and abdominal muscle stretching (unable to maintain former posture), leading to more weight to be supported by lower back [9]. Gravity centre theory indicates that, during pregnancy, body centre of gravity shifts to the front, causing core muscles to work harder in keeping the stability of the lumbar backbone and pelvic girdle [10]. Yoga is a type of non-pharmacological therapy that can reduce the pain [11]. Body poses in yoga can help flex muscles surrounding the backbone, which will help alleviate pain and improve body capability to move [12].

In this research, One-Way Anova test on the level of back pain experienced by pregnant women indicates significant difference on the average level of back pain of the four groups. This is confirmed by $p$-value $=0,001<\alpha$ (Table 2). Furthermore, dual comparison test using LSD test indicates a significant difference on the average level of back pain experienced by the control group and treatment group, especially the treatment group receiving combined treatment of prenatal yoga and prenatal aromatherapy massage. The average value indicates a less pain experienced by the treatment group compared to the control group. This means combined treatment of prenatal yoga and aromatherapy massage is more effective in alleviating back pain than prenatal workout for the control group.

Practicing prenatal yoga for 20 minutes once a week for four weeks has a positive impact on pregnant women [13]. Pregnant women who practice yoga experience a reduced pain in the lower back [14]. Aromatherapy massage can significantly decrease stress and improve the immune system 
of pregnant women. Massage is given to encourage comfort, maintain overall body function, provide emotional support, and alleviate back pain [15].

As elaborated previously, there has not been any research attempting to determine the direct connection between combined treatment of prenatal yoga and aromatherapy massage with the level of beta endorphin in pregnant women. Massage can reduce pain intensity and increase the level of beta endorphin. Increasing level of beta endorphin on pregnant women will disrupt the transmission of pain by increasing the circulation of neurotransmitter produced to the neural synapses in the central nervous system. Endorphin binds with presynaptic membrane and hinders the release of chemicals as well as prevent the spreading, alleviating the pain [16].

The result of one-way Anova Test on the level of beta endorphin indicates a significant difference in the average level of beta endorphin from all four observed sample groups as shown by p-value $=0,04<\alpha$ (Table 3 ). Additionally, dual comparison test using LSD test indicates a significant difference on the average level of beta endorphin of the control group and treatment group, especially the treatment group receiving combined treatment of prenatal yoga and prenatal aromatherapy massage. The average value indicates a higher decrease of pain experienced by treatment group compared to the control group. This proves that the combined treatment of prenatal yoga and aromatherapy massage can better increase the level of beta endorphin on the treatment group, as opposed to the control group.

The result of this study is consistent with McCullough study, that elaborates that 61 out of 90 pregnant women (divided into 6 groups) receiving reflexology treatment see pain frequency decreasing by as much as 1,63 points and an increase of beta endorphin level by $15,18 \%$ (reflexology group) [17]. Massage is a relaxation technique designed to alleviate pain by reducing the secretion of adrenaline and noradrenaline, leading to increased endorphin. Additional to massage, yoga is also effective for improving the level of beta endorphin [18], reduce psychological stress, anxiety, and depression during pregnancy by triggering the release of endorphin, which is biological endogenous opioid system serving as the pain modulator [19].

Beta endorphin and cortisol are two hormones which are directly correlated with stress and pain responses. Both hormones are synthesized in the hypothalamus-pituitaryadrenal (HPA) cortex as the release of these hormones trigger the release of corticotrophin $(\mathrm{CRH})$, leading to the secretion of proopiomelanocortin glands (POMC) by the pituitary gland. The cleaving of POMC triggers the production of Adrenoorticotropin (ACTH) in the pituitary, stimulating the production of cortisol in the adrenal cortex and the release of beta endorphin in the anterior-pituitary gland. Cortisol is released as a response to chronic stress, physiological condition, and psychological condition, for instance pain and fear. Physiological effect from stress causes the increase in cortisol level, lower pain threshold, and faster heart rate. The correlation between cortisol level and body fluids (such as plasma, blood, urine, and saliva) has been documented in the general population and pregnant women. Beta endorphin is released into the blood stream from the pituitary gland as a response to an episode of acute pain and stress, and it helps alleviate pain, lower pain perception, and increase pain threshold. The concentration of beta endorphin typically is examined from blood, plasma, or cerebrospinal fluid (CSF) [17].

\section{CONCLUSION}

According to the research result and discussion above, it is conclusive that the combined treatment of prenatal yoga and prenatal aromatherapy massage has a significant impact in the difference of average level of back pain and beta endorphin level at $p$-value $<0,05$. This means the combination of prenatal yoga and prenatal aromatherapy massage treatments for pregnant women can alleviate back pain and increase the level of beta endorphin when compared to the single treatment group and control group.

\section{REFERENCES}

[1] Saudia. B.E.P. and Sari O.N.K. The Difference Of Endorphin Massage With Warm Compresses To Reduce Back Pain in Trimester Third Pregnant Women in Public Health in the working area of Mataram City Indonesia. Prime Health Journal. 2018; 12 (1):23-29

[2] Abino. J and Graver.J.N. Pregnancy and Low Back Pain. Current Reviews in Musculoskeletal Medicine. 2008; 1(2):pp 137-141.

[3] Shetty. H., Biliangady. H., Kumar.D. Efficacy of Yoga on Low Back Pain and Disability in Primigravidas. International Journal of Physiotherapy. 2016; 4:182-185.

[4] Valdiglesias V. Maseda.A. Lorenzo-Lhophez.L., Pasaro.E., MillonCalenti.J.C., et al. Is Salivary Chromogranin-A a Valid Psychological Stress Biomarker During Sensory Stimulation in People with Advanced Dementia?.Journal Of Alzheimer's Disease. 2017; 55(4):1509-1517.

[5] Steel. A and Adams. J. The Role Of Naturopathy in Pregnancy, Labour and Postnatal Care: Broadening The Evidence-Base. Complement. Ther. Clin.Pract. 2011;17(4):189-192.

[6] Aprilia. Y and Ritchmound.B. 2011. Gentle Birth : Comfortable Childbirth without Pain. Jakarta : PT. Gramedia Widiasarana Indonesia

[7] Devi M, Sulaiman, Rosnaini, Jawiah. The Effect of Antenatal Yoga on Reducing Complaints of Third Trimester Pregnant Women. Medical and Health journal, 1(1): 47-53.

[8] Muhimah.N.A and Safei. 2010. Complete guide to healthy exercise specifically for Pregnant Women. Yogyakarta: Power Book.

[9] Hughes. C.M., Liddle.S.D., Sinclair.M., Mc.Cullough.J.E.M. The use of Complementary and Alternative Medicine (CAM) for pregnancy related low back and or pelvic girdle pain : An online survey. Journal Complementary Therapies in Clinical Practice. 2018; 31:379-383.

[10] Chiu-Fang Yan MS., Kuan-Chia Lin. Effect of A stability Ball Exercise Programme on Low Back Pain and Daily Life Interference During Pregnancy. Midwifery Journal. 2014; 30(4): 412-419.

[11] Shindu, P. Yoga for A Healthy, Happy, and Meaningful Pregnancy, Fit Series. Bandung: Qonita, Mirzan Pustaka. 2009; 50-98.

[12] Kusaka. M., Matsuzaki. M., Shiraishi. M., Haruna. M. Immediate Stress Reduction Effect of Yoga During Pregnancy : One group PrePost Test. Women and Birth Journal. 2016; 29(5):82-88.

[13] Timlin D, Simpson EEA. A preliminary Randomized Control Trial of The Effects of Drug Yoga on Psychological Well-Being in Northern Irish First Time Mothers. Midwife, An International Journal. 2017.

[14] Resmi DC, Saputro S.H, Runjati. The effect Of Yoga on Lower Back Pain in Third Trimester Pregnancy at Kalikajar I Public Health Centre Wonosobo District. Health Scientific Journal. 2017.

[15] Chen, P.-J., Chou, C.-C., Yang, L., Tsai, Y.-L., Chang, Y.-C and Liaw, J.-J. Effects of Aromatherapy Massage on Pregnant Women's Stress and Immune Function: A Longitudinal, Prospective, Randomized Controlled Trial. The Journal of Alternative and Complimentary Medicine. 2017; 23(10): 778-786.

[16] Ariyani Y, Masrul, Evareny L. Influence of Back Massages on the intensity of pain in the first stage of the latent phase of normal labour through increased levels of endorphins. Andalas Health Journal, 2015. 
[17] Mc Cullough. J.E.M., Liddle.S.D., Close.C., Sinclair.M., and Hughes.C.M. Reflexology: A randomized controlled trial investigating the effects on beta-endorphin, cortisol and pregnancy related stress. Complementary Therapies in Clinical Practice. 2018; 31: 76-84.

[18] Bolbol-Haghighi N, Masoumi SZ, Kazemi F. Effect of Massage Therapy on Duration of Labour: A Randomized Controlled Trial.
Journal of Clinical and Diagnostic Research:JCDR. 2016;10(4):QC12-QC15.

[19] Suri, M., Sharma, R. and Saini.N. Review Article NeuroPhysiological Correlation Between Yoga, Pain, and Endorphins. International Journal of Adapted Physical Education and Yoga. 2017:2(9):18-32. 